\title{
Learning about occlusion: Initial assumptions and rapid adjustments
}

\author{
Olga Kochukhova *, Gustaf Gredebäck \\ Department of Psychology, Uppsala University, 75142 Uppsala, Sweden
}

Received 29 January 2006; revised 17 August 2006; accepted 18 August 2006

\begin{abstract}
We examined 6-month-olds abilities to represent occluded objects, using a corneal-reflection eye-tracking technique. Experiment 1 compared infants' ability to extrapolate the current pre-occlusion trajectory with their ability to base predictions on recent experiences of novel object motions. In the first condition infants performed at asymptote $(\sim 2 / 3$ accurate predictions) from the first occlusion passage. In the second condition all infants initially failed to make accurate prediction. Performance, however, reached asymptote after two occlusion passages. This is the first study that demonstrates such rapid learning effects during an occlusion task. Experiment 2 replicates these effects and demonstrates a robust memory effect extending $24 \mathrm{~h}$. In occlusion tasks such long-term memory effects have previously only been observed in 14-month-olds (Moore \& Meltzoff, 2004).
\end{abstract}

(c) 2006 Elsevier B.V. All rights reserved.

Keywords: Infants; Occlusion; Extrapolation; Rapid learning, Gaze tracking

\section{Introduction}

Since the first studies of Piaget (1952), large numbers of investigations have focused on infants' developing abilities to represent occluded objects (for example,

\footnotetext{
This manuscript was accepted under the editorship of Jacques Mehler.

* Corresponding author. Fax: +46184712123.

E-mail address: olga.kochukhova@psyk.uu.se (O. Kochukhova).
} 
Baillargeon, 1999; Mareschal, 2000; Spelke, Breinlinger, Macomber, \& Johnson, 1992; Spelke, Katz, Purcell, Ehrlich, \& Breinlinger, 1994; Wilcox, Rosser, \& Nadel, 1994). In a set of pioneering studies, 5- and 9-month-old infants were presented with a toy train that moved through a tunnel (Meichler \& Gratch, 1980; Nelson, 1971, 1974). The results indicated that 5-month-old infants failed to anticipate the reappearance of the train on initial trials while 9-month-olds extrapolated the motion of the train from the first trial. Five-month-olds could, however, learn to predict the reappearance of the objects after several trials. These early reports demonstrate that there are at least two different ways in which infants can predict the reappearing object; infants can either extrapolate the pre-occlusion trajectory or base their predictions on recent experiences accumulated over successive trials. Subsequent studies of infants' representational abilities have continued to investigate these two modes of prediction (Gredebäck \& von Hofsten, 2004; Johnson, Amso, \& Slemmer, 2003; Moore \& Meltzoff, 2004; Rosander \& von Hofsten, 2004; Spelke et al., 1992; von Hofsten, Feng, \& Spelke, 2000).

Some research has focused on infants' tendency to extrapolate the motion of temporarily occluded objects. For instance, Spelke et al. (1994) presented 4-, 6-, $8-$, and 10-month-old infants with objects moving on linear trajectories that either continued to move along this trajectory in absence of an obstruction (possible event), or moved through an obstacle positioned behind an occluder (impossible event). Six-month-old infants increased their looking times to the impossible event; suggesting that infants expected the moving object to continue on a connected and unobstructed path.

Infants' abilities to extrapolate have also been investigated using a head tracking paradigm. In two studies by Spelke and von Hofsten 6-month-old infants were presented with toys moving on diagonal paths behind an occluder (Spelke \& von Hofsten, 2001; von Hofsten et al., 2000). On half the trials the toy emerged along the linear extension of the pre-occlusion trajectory (linear trials). During remaining trials the toy shifted its trajectory behind the occluder by $90^{\circ}$; continuing downwards on an orthogonal path (non-linear trials). The authors found that infants quickly learned to extrapolate the linear extension of the occluded trajectory; providing support for an initial tendency to base predictions on an extrapolation of the pre-occlusion trajectory. These 6-month-olds, however, also learned to predict the non-linear trajectories given substantial experience.

Recently, Rosander and von Hofsten (2004) presented horizontally moving objects to 4-month-old infants. By measuring how infants tracked the disappearing object with their gaze, the authors were able to conclude that infants fixated the reappearance side of the occluder before the object emerged; thus following the linear continuation of object's trajectory. This occurred even in trials where the object turned behind the occluder and reappeared at the entrance side, suggesting that infants extrapolated the pre-occlusion trajectory of the object. Similar abilities to extrapolate pre-occlusion trajectories have been demonstrated using circular trajectories (Gredebäck \& von Hofsten, 2004; Gredebäck, von Hofsten, $\&$ Boudreau, 2002), indicating that infants extrapolate curvilinear trajectories in a similar manner. 
Other occlusion studies have stressed infants' abilities to predict future events based on more long-term experiences. Among others, Moore and Meltzoff (2004) demonstrated that 14-month-olds could represent the location of a hidden toy and retain this information over a $24-\mathrm{h}$ period; recovering the toy from the correct location as they came back to the lab on the following day. Furthermore, (Johnson et al., 2003) provided evidence that 4-month-old infants improved their performance on an occlusion task if they were trained with fully visible trajectories. Infants also improved their performance over successive trials (Rosander \& von Hofsten, 2004).

Outside the occlusion paradigm numerous studies have demonstrated rapid and stable learning effects in infants. For example, Canfield and Haith (1991) and Haith (1994) used saccade latencies to demonstrate that 2-month-old infants could predict sequences of pictures that alternated between two locations. Other work in visual memory that does not involve occlusion events suggests that infants have substantial long term memory for events. Rovee-Collier and colleagues (for review, see Hartshorn \& Rovee-Collier, 1997; Hartshorn et al., 1998; Hitchcock \& Rovee-Collier, 1996) used operant conditioning to access 2to 6-month-old infants' ability to associate their own kicking with the motions of a mobile. The results of these studies showed that from 2 month of age infants could show retention for the task for 1 day and 6-month-olds could show retention for 14 days.

In conclusion, based on earlier research it can be suggested that there are at least two different ways in which infants predict the future trajectory of temporarily occluded objects. First, infants can base their predictions of where and when objects will reappear on a general assumption of how objects move. For example, an object that moves on a straight trajectory will continue to move on that trajectory (extrapolations). Second, infants can base their predictions of upcoming object motions on recent experience with moving objects. In other words, infants can form their predictions of how future events unfold on the assumption that resent events will repeat themselves in similar contexts.

All above mentioned occlusion studies have presented infants with multiple repetitive trials (Gredebäck \& von Hofsten, 2004; Gredebäck et al., 2002; Johnson et al., 2003; Meichler \& Gratch, 1980; Nelson, 1971, 1974; Rosander \& von Hofsten, 2004; Spelke \& von Hofsten, 2001; von Hofsten et al., 2000) that allowed infants to predict the reappearance location from the current pre-occlusion trajectory and to accumulate experience over time.

No studies have ever, to our knowledge, disentangled the two predictive mechanisms by presenting infants with sets of stimuli that can only be predicted through linear extrapolations or through recent experience of similar events. There are multiple benefits to pursuing such a research strategy. First of all, this gives us the opportunity to investigate the above defined separation between extrapolations and recent experiences during occlusion tasks. Second, it allows us the opportunity to closely examine how each of these processes progress over time; looking at experience based predictions without interference from initial successful extrapolations and vice versa. 


\section{General method}

\subsection{Stimuli and apparatus}

Gaze was measured with a Tobii 1750 eye tracker with an infant add-on (www.Tobii.se). The system records the reflection of near infrared light in the pupil and cornea of both eyes at $50 \mathrm{~Hz}\left(\right.$ accuracy $=0.5^{\circ}$, spatial resolution $=0.25^{\circ}$ ) as the participant watches an integrated 17 inch monitor. During calibration a blue and white sphere (Gredebäck \& von Hofsten, 2004) expanded and contracted (extended diameter $=3.3^{\circ}$ ) in synchrony with a sound.

The experimental stimuli consisted of a bird's view of a scene containing a multicolored ball (radius $=1^{\circ}$ ) rolling on a blue background $\left(24^{\circ} \times 28^{\circ}\right)$. In the middle of the scene a wooden textured occluder (radius $=3.5^{\circ}$ ) covered the ball as it rolled underneath. Both the ball and the occluder cast light shadows on the background and the ball reflected some light; this was done to enhance the perception of a three-dimensional occlusion event (see Fig. 1).

The specific trajectories being presented in each condition are described in the method sections of each experiment below. There are, however, commonalities between all trajectories that will be addressed here. Each experimental session included 16 complete occlusion passages, in which the ball rolled back and forth between two endpoints (velocity $=5^{\circ} / \mathrm{s}, 0.167 \mathrm{~Hz}$, motion amplitude $=22^{\circ}$ ). During each of these occlusion events the ball was fully covered by the occluder (time out of sight) for $700 \mathrm{~ms}$. The duration of accretion/deletion, when the ball was partly covered by the occluder, lasted $333 \mathrm{~ms}$.

The 16 complete occlusion events were presented in pairs. All in all infants were presented with eight movies each containing two complete occlusion events. The total duration of each movie equaled $9000 \mathrm{~ms}$. In every movie the object always started to move from behind the occluder $(0 \mathrm{~ms})$ and its initial motion was directed in one of four directions (up, down, left, or right). After $1500 \mathrm{~ms}$ the ball reversed its direction moving towards the occluder, completing its first full occlusion passage. After $4500 \mathrm{~ms}$ of the movie the ball once more reversed its direction, moving back
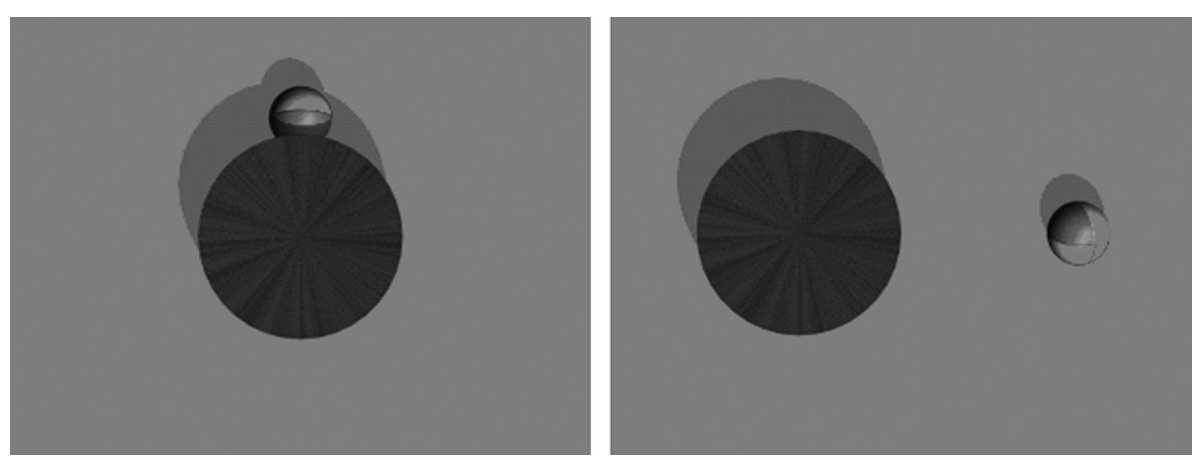

Fig. 1. Close-ups of the stimuli. 
behind the occluder. Following this second full occlusion passage, the ball reversed its direction for the last time (at $7500 \mathrm{~ms}$ ), stopping once it reached the center of the screen at $9000 \mathrm{~ms}$ (behind the middle of the occluder).

In each movie the ball was completely covered for $2100 \mathrm{~ms}$. This includes two complete occlusion events $(2 \times 700 \mathrm{~ms})$ plus the initial and final phase of each movie; in these phases the ball emerged and disappeared behind the occluder (being completely covered for $2 \times 350 \mathrm{~ms}$ ).

All in all, each infant perceived 16 complete occlusion passages. As a consequence thereof infants could contribute a maximum of 16 data points during one experimental session. Examples of the trajectories used can be found in Section 3.1.

\subsection{Procedure}

Families with children of appropriate age were contacted with a letter describing the study and an invitation to participate. If they decided to do so, an appointment was made. Once in the lab each family was provided with a verbal description of the study, its purpose, and the methods used. The parents signed a consent form before the study began. The study was approved by the ethics committee of the Research Council in the Humanities and Social Sciences and therefore in accordance with the ethical standards specified in the 1964 Declaration of Helsinki.

Infants were seated in a safety car seat that was placed on the participating parent's lap in front of the Tobii eye tracker and monitor (eyes approximately $60 \mathrm{~cm}$ from the screen). Before the experiment a calibration procedure was carried out. In preparation for the calibration the experimenter adjusted the eye tracker to make sure that the reflections of both eyes were centered in the cameras field of view.

During calibration a blue and white ball appeared on each of 9 calibration points in a random order. If infants' attention was drawn to other parts of the visual field during this procedure the experimenter could present alternative movies with novel sounds at the current location. At the end of the calibration a graph appeared that reported how successful the calibration was; any unsuccessfully calibrated points were recalibrated. In each condition infants were presented with one session of 8 stimuli movies each containing two full occlusion passages. In between movies infants were presented with one of 8 different attention grabbing movies (horizontal and vertical extension $=5.7^{\circ}$ ) displaying a small toy that moved and sounded in the middle of the screen. Each experimental session lasted no more than $7 \mathrm{~min}$.

Following the final movie each family was shown a recording that combined the stimuli with infant's gaze. This provided an opportunity for the parents and the experimenter to discuss the study and the infants' reactions to the occlusion events. As compensation for their participation each family received either two movie tickets or a gift certificate of a $\mathrm{CD}$; both with a total value of $12 €$.

\subsection{Data analysis}

The Tobii eye tracker stored coordinates of gaze from both eyes, together with a measurement of data quality (five point scale). Gaze was calculated based on both 
eyes in most instances and only from one eye in those instances were the eye tracker data quality was poor for the other eye. All analysis was performed using custommade analysis programs in the Matlab (Mathworks Inc.) environment.

The area surrounding the edge of the occluder was divided into four areas (top, down, left, and right side of the occluder); each covering $90^{\circ}$ of the occluder edge. These areas extended both inside and outside the occluder, covering all but the final $2^{\circ}$ near the occluder center and extending outwards to cover the entire amplitude of the targets motion (see Fig. 2).

To be included in the analysis infants had to attend to at least half of all (4/8) stimuli movies. During these movies infants had to attend to at least one occlusion passage, defined as an occluder event in which the infant tracked the target for at least $200 \mathrm{~ms}$ prior to the disappearance of the ball. In addition, infants had to fixate the reappearance location before the target emerged there or continue to track the target after its reappearance for an equal amount of time.

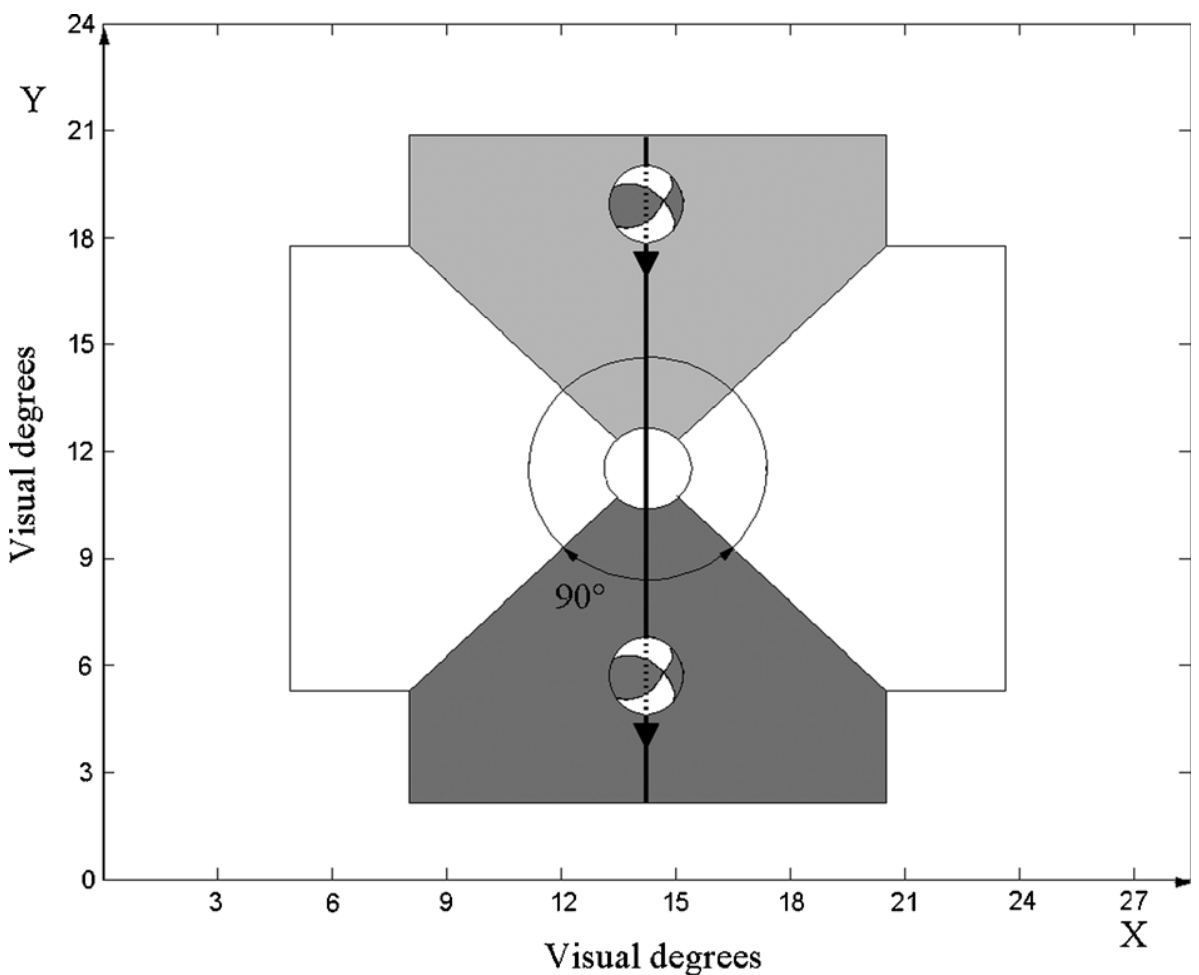

Fig. 2. The different areas covered by the analysis during an example trajectory. The straight vertical line depicts the current trajectory being analyzed and the outer circle indicates the location of the occluder. The four large areas (not including the area covered by the inner circle) define the four possible reappearance locations of the target. The light gray area represents the disappearing edge and the dark grey area the reappearance edge. 
First of all, infants' response to each occlusion passage was dichotomized as either predictive or reactive. During reactive responses gaze moved across the occluder after the ball had been visible for $200 \mathrm{~ms}$. Predictive responses included all passages in which gaze moved to any of the three remaining areas (not counting the area in which the ball disappeared) before the ball had been visible for $200 \mathrm{~ms}$. This criterion was based on the average reactive saccadic latency to moving targets in adults (Engel, Anderson, \& Soechting, 1999) and infants (Gredebäck, Örnkloo, \& von Hofsten, 2006). It has previously been used to distinguish reactive from predictive saccades in occlusion tasks (Gredebäck \& von Hofsten, 2004; Gredebäck et al., 2002; Rosander \& von Hofsten, 2004).

Two primary measurements of infants' performance were used in the current paper. In both of them each data point was dichotomized in terms of success or failure. Both analyses were based on percentage scores calculated separately for each infant. Prediction rate reports how often infants succeed in predicting the reappearance of the target relative to the total number of attended passages (number of predictive responses/number of predictive and reactive responses). Note that this measurement focuses on the timing of infants gaze shift over the occluder; it does not take into account where along occluder edge infants predicted the target to reappear.

Whether infants fixated the correct reappearance location of the target or not is measured through accuracy rate (number of accurate predictions/number of accurate and inaccurate predictions). This measurement reports whether infants predictive responses terminated near the actual reappearance location of the target (as defined by the dark grey area in Fig. 2) or at some other part of the occluders edge (white areas in Fig. 2). The disappearance edge of the occluder is not included in this measurement as infants have to move gaze from this location in order to continue tracking the target.

In addition, each session was analyzed using both linear and curvilinear regression analysis. This was done in order to see if infants' performance changes over successive occlusion passages. The regression line with the highest explained variance (linear or curvilinear) was reported and displayed in the figures below.

\section{Experiment 1}

The first experiment compares infants' ability to extrapolate trajectories with their ability to base predictions on recent experience from previous trials. The ability to extrapolate a trajectory relies on the assumption that the pre-occlusion trajectory will continue in the same direction behind the occluder, not on memories of recent events. By presenting infants with multiple linear trajectories whose starting position (and thereby disappearance and reappearance locations) were randomized between movies an estimate was obtained of infants ability to extrapolate trajectories of occluded objects, independently of recent experiences. This condition is refereed to as the variable linear condition.

In a similar manner, pure memory based predictions can only be isolated if infants are presented with repetitive non-linear trajectories in which the reappearance location 
cannot be predicted through extrapolations. Following this line of reasoning a second group of infants were presented with multiple identical trajectories that suddenly turned by $90^{\circ}$ behind the occluder. This condition is referred to as the fixed non-linear condition. No previous studies have presented infants with non-repetitive variable linear trajectories. Neither has there been any study that has used repetitive and fixed nonlinear trajectories in isolation, without intervening blocks of linear trajectories.

\subsection{Method}

\subsubsection{Participants}

Each family visited the lab on one occasion in close proximity to the infant's 6month birthday. A total of 46 infants participated in Experiment 1. Twenty-one infants ( 13 boys and 8 girls; mean age $26: 5$ weeks, $S D=3.2$ ) took part in the variable linear condition. Of these, two infants ( 1 boy and 1 girl) were excluded from analysis. In the fixed non-linear condition 25 infants (14 boys and 11 girls; mean age 26:4 weeks, $S D=2.6$ ) participated and four of them ( 1 girl and 3 boys) were also excluded from the following analysis. All excluded infants attended to less than half of the experimental movies. Thus, 40 infants were analyzed in Experiment 1; 19 in the variable linear condition and 21 in the fixed non-linear condition.

\subsubsection{Stimuli and design}

As mentioned above, participants in Experiment 1 were presented with one of two conditions. Each infant either participated in the variable linear or the fixed non-linear condition.

In the variable linear condition infants were presented with eight movies (including 16 occlusion passages) randomly sampled from a set of four movies (the differences defined by the four possible starting directions of the object's linear motion). One example of a linear trajectory can be observed in Fig. 3A. In this example the ball appeared next to the lower edge of the occluder, moving downwards. After reaching the lower edge of the screen it changed direction by $180^{\circ}$ to move behind the occluder. The ball then reappeared on the opposite edge of the occluder, reached the upper edge of the screen and then changed by $180^{\circ}$ to move back along the same trajectory behind the occluder one last time before disappearing behind the occluder at the end of the movie.

The individual stimuli presented to infants in the fixed non-linear condition were identical with the stimuli in variable linear condition with one exception. During each occlusion passage the ball did not continue along the linear extension. Instead its direction of motion shifted $90^{\circ}$ to a novel trajectory perpendicular to the original direction. For each of the two complete occlusion passages of each experimental movie the ball reversed its direction, oscillating back and forth between two end points. All in all, there were eight different trajectories (the ball could start out in either of four directions up, down, left, and right and the shift $\pm 90^{\circ}$ to either of two directions). Each infant was presented with only one of these trajectories, where the ball oscillated between the same two endpoints throughout the session. The choice of the trajectory presented to an infant was determined by randomising. Thus, 
A
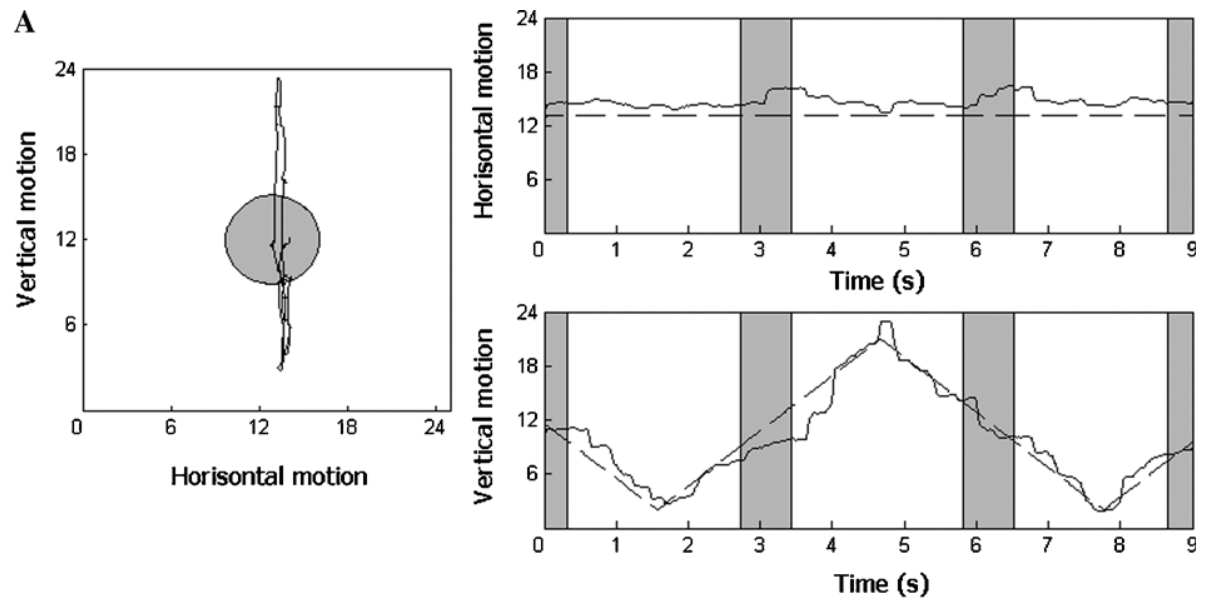

B
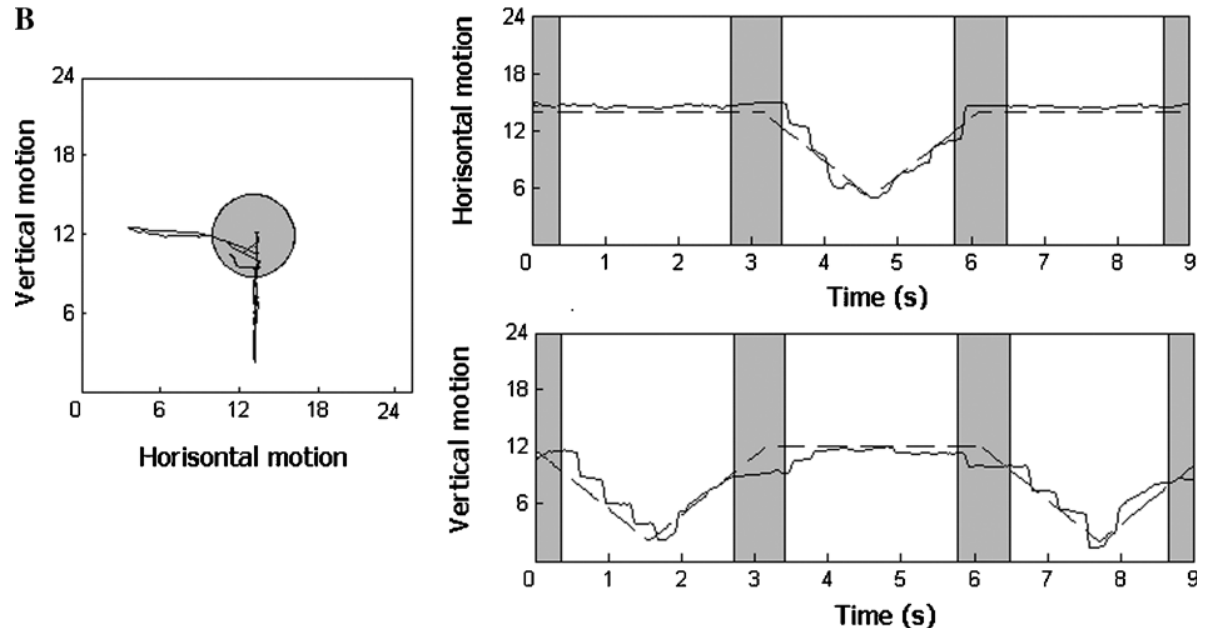

Fig. 3. Examples of the targets' trajectory and gaze in one linear (A) and one non-linear (B) movie. Grey areas indicate the location of the occluder. The solid line represents gaze and dashed line represent the target. Note: The first passage is reactive and second passage is predictive in both conditions.

each infant only saw one and the same non-linear trajectory throughout the entire session. Fig. 3B displays a trajectory that starts of moving downwards turning $90^{\circ}$ to the left behind the occluder.

\subsection{Results}

\subsubsection{Overall performance}

A total of 221 passages $(73 \%)$ were included in the analysis of the variable linear condition whereas 219 passages $(65 \%)$ were included in the fixed non-linear condition. 
Remaining passages did not meet the inclusion criteria, meaning that infants did not fixate or track the target for $200 \mathrm{~ms}$ both prior to and following the occlusion event.

To provide an overall perspective on infants' global performance we categorized their behavior into three types of responses (prior to reporting prediction and accuracy rates below). On most passages infants fixated the disappearance edge of the occluder for the entire duration of time spanning from the time when the object became completely occluded until infants made a predictive or reactive saccade ( $76 \%$ of all attentive passages in both the variable linear and fixed non-linear condition). On other passages infants moved from the disappearance edge to fixate the centre of the occluder for a while before making their predictive or reactive saccade (19\% and $20 \%$ of all attentive passages in the fixed non-linear and variable linear condition). The third category included alternative search patterns, such as looking around the scene and/or quickly scanning the occluder edge without clear fixations ( $5 \%$ and $4 \%$ of all attentive passages in the non-linear and linear conditions). ${ }^{1}$

The median predictive saccade occurred after the ball had been occluded for $472 \mathrm{~ms}(S E=34 \mathrm{~ms})$ in the variable linear condition and after $627 \mathrm{~ms}(S E=34 \mathrm{~ms})$ in the fixed non-linear condition. During reactive passages infants waited $1033 \mathrm{~ms}$ $(S E=7 \mathrm{~ms})$ during linear and $1023 \mathrm{~ms}(S E=6 \mathrm{~ms})$ during non-linear passages (complete occlusion duration $=700 \mathrm{~ms}$ ).

The overall prediction rate (number of predictive responses/number of predictive and reactive responses) did not differ between the two conditions. Infants' prediction rate equaled $0.43(S E=0.021)$ during the variable linear condition and 0.47 $(S E=0.037)$ during the fixed non-linear condition. In a similar manner, the overall accuracy rate (number of accurate predictions/number of accurate and inaccurate predictions) remained equal. The portion of accurate predictions was $0.66(S E=0.07)$ during non-linear passages and $0.76(S E=0.025)$ during linear passages.

There are, however, statistically significant differences between infants' accuracy rate at the first two occlusion passages $(t(34)=2.7, p<.01)$. Later trials did not differ between the two conditions. In the variable linear condition infants' accuracy rate did not change over successive occluder passages $(0.76 ; S E=0.025)$ whereas infants presented with the fixed non-linear condition quickly improved their accuracy rate from 0.0 on the first occlusion passage to 0.63 on the third passage. This difference between the two conditions is thoroughly described in the section below.

\subsubsection{Within session analysis of the variable linear condition}

When presented with multiple linear trajectories infants made predictive saccades to the correct reappearance location in $62 \%$ of cases from the first passage and continued to do so at the same level over the entire session. Thus, the accuracy rate equalled 0.62 on the first occlusion passage, 0.75 during the second passage and 0.67 during the third passage (the accuracy rate during the final 16th passage equalled 0.75). A passage by passage regression analysis of performance did not demonstrate

\footnotetext{
${ }^{1}$ Looking away from screen resulted in missing data; such performance is therefore not included in these categories.
} 

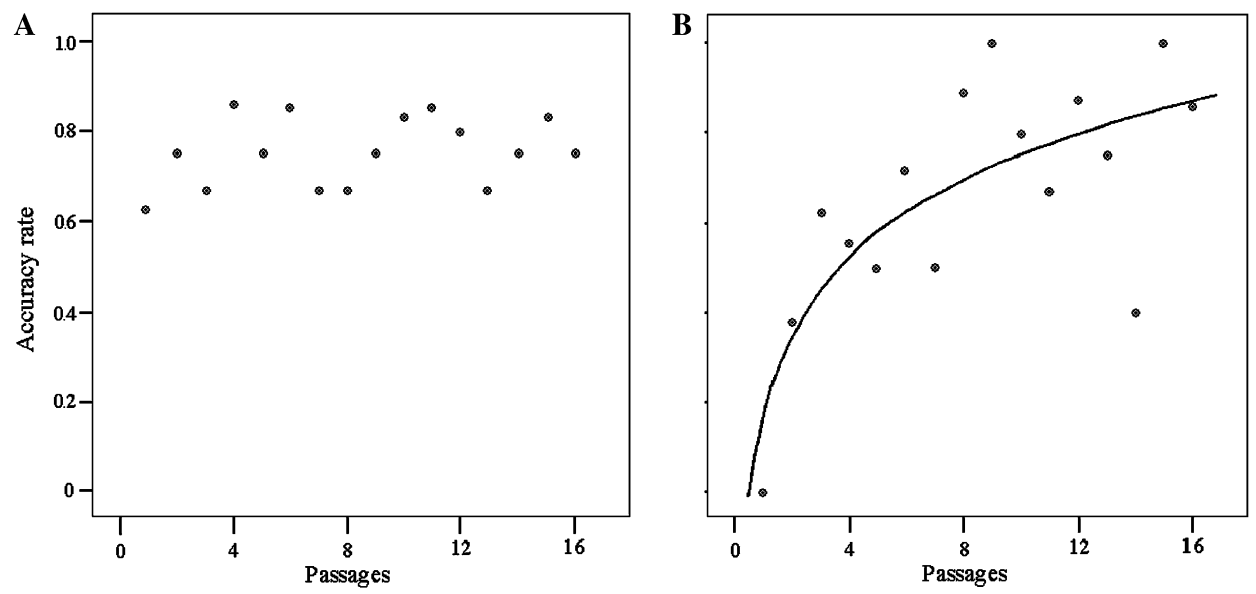

Fig. 4. Accuracy rates (number of accurate predictions/(number of accurate + inaccurate predictions)) over successive occlusion passages in the variable linear (A) and fixed non-linear (B) conditions of Experiment 1. $X$-axis shows each occlusion passage during one experimental session. The solid curve depicts the regression line with most explained variance. Note: no significant regression line exists for the linear condition.

improvements in infants' performance in neither prediction rate nor accuracy rate over successive occlusion passages. Fig. 4A shows that infants' accuracy rate or ability to extrapolate linear trajectory stays on the same level during the entire experimental session.

Remaining incorrect predictive saccades ( 1 - accuracy rate $)$ were evenly distributed between the two remaining areas covering the occluder (see white areas in Fig. 2).

\subsubsection{Within session analysis of the fixed non-linear condition}

In response to the fixed non-linear condition the accuracy rate changed dramatically from 0.0 during the first occlusion passage to 0.37 during the second passage and 0.63 during the third passage (the accuracy rate during the last passage equalled 0.86). A passage by passage regression analysis revealed a strong increase in accuracy rate over successive presentations. In other words, the more times infants saw the ball disappear and reappear behind occluder the more accurate they were in predicting where the ball will reappear next time. Most variance was explained by the equation ${ }^{2}: y=17.3+25.2 \ln (x)$ demonstrating that the proportion of predictive gaze shifts made to the correct reappearance location increased during the session according to a logarithmical function and that most improvement in accuracy rate occurred during the first few passages, $R^{2}=.45$, $F(1,14)=11.37, p<.005$. The regression line and underlying accuracy rates can

\footnotetext{
${ }^{2}$ Where $x$ - proportion of accurate predictive gaze shifts made at every complete occlusion and $y-$ regression function, describing increase of accuracy rate over session.
} 
be observed in Fig. 4B. No differences were observed for prediction rate over successive presentations.

Incorrect predictive saccades, that terminated within another area than the actual reappearance location of the ball (34\% of all predictions) were strongly biased towards the linear extension of the targets pre-occlusion trajectory during non-linear passages (One-Way Goodness of Fit $\chi^{2}$ ), $\chi^{2}(1)=8.26, p<.05$. Twenty-five percent of all predictions were directed to this location and only $9 \%$ to the remaining location (note that the ball never appeared in either of these two locations). The temporal distribution of the tendency to make linear extrapolations in the fixed non-linear condition was analysed separately (see below).

\subsubsection{Extrapolations in the fixed non-linear condition}

Linear extrapolations were observed in the beginning of the fixed non-linear condition. In the first passage $100 \%$ of all predictive gaze shifts were made as linear extrapolations whereas the second passage included only $37.5 \%$ extrapolations. This tendency was best represented by the logarithmic function $y=64.5-19.75 \ln (x)$ visible in Fig. 5, describing a rapid decrease in the tendency to extrapolate the pre-occlusion trajectory over the first few passages, $R^{2}=.34, F(1,14)=7.1, p<.02$.

\subsection{Discussion}

Experiment 1 demonstrates two different ways in which 6-month-old infants can predict where and when an occluded target will reappear. Infants will

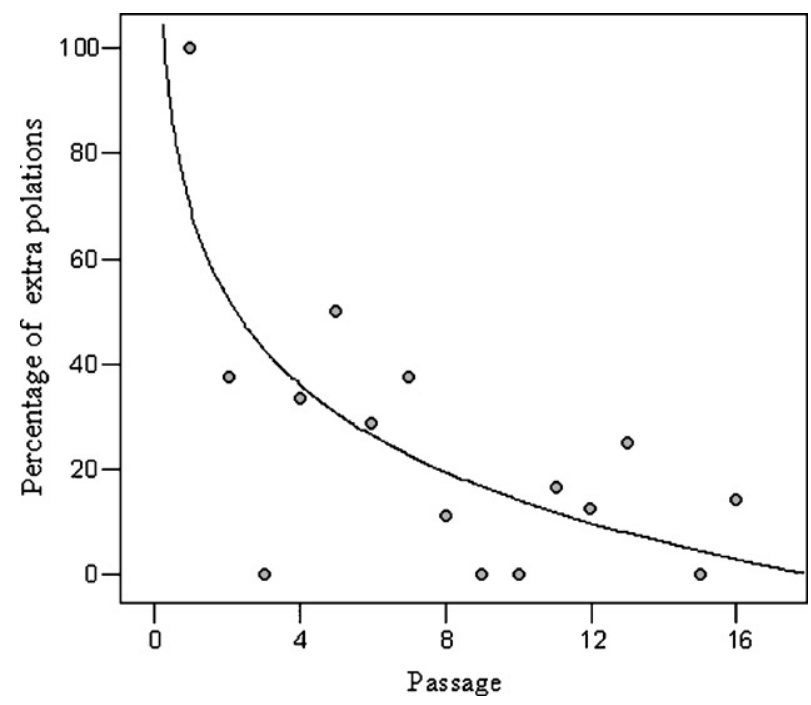

Fig. 5. Percentage of extrapolations during the predictive gaze shifts (i.e. inaccurate predictive gaze shifts that followed the linear extension of the target's pre-occlusion trajectory) during the fixed non-linear condition of Experiment 1. The solid line represents the regression line with most explained variance. 
extrapolate unknown trajectories and will continue to do so unless alternative processes become available to guide predictions. However, once a repetitive pattern appears in the stimulus array infants will use this information to form expectations that accord with this experience. Such memory based predictions are formed rapidly and have reached asymptote by the third occlusion event. The initial tendency to extrapolate and the ability to base predictions on recent experiences are discussed separately below.

Six-month-olds will assume that objects moving on linear trajectories prior to an occlusion event will continue accordingly. Infants' initial reliance on extrapolations is equally prominent in both conditions. In the variable linear condition, infants' performance remained unchanged throughout the entire session whereas the tendency to extrapolate the pre-occlusion trajectory quickly weakened in the fixed non-linear condition. The fact that infants' initial predictions were maintained in one condition and diminished in the other is most likely caused by an interaction between two factors; whether initial extrapolations are successful or not and whether alternative information (in this case repetitive trajectories) was available to guide future predictions. If initial extrapolations accurately predict where the target will reappear, infants will continue to extrapolate the pre-occlusion trajectory, thus maintaining the initial asymptotic performance. However, if the same extrapolations provide an unreliable predictor of future outcomes then infants rapidly learn to rely on alternative sources of information.

The rapid shift from extrapolations to a reliance on recent experiences in the fixed non-linear condition occurred mainly during the first two occlusion passages (see Fig. 5); an effect that is much more rapid than what has previously been reported (von Hofsten et al., 2000). The fact that von Hofsten et al. reported a much slower shift from linear to non-linear predictions is likely due to design differences between the two studies. In the study by von Hofsten et al., infants were presented with blocks of linear repetitive trials followed by non-linear repetitive passages. This means that infants had to inhibit a more repeated response, similar differences following repetitions have been demonstrated using the A, not B paradigm (Munakata, 1998; Piaget, 1952). In addition, the current study measures eye movements whereas von Hofsten et al. relied on head movements as a dependent measure. It is possible that the two measurements have slightly different lags, resulting in a more rapid shift from extrapolations to memory based predictions in the current study compared to von Hofsten et al. (2000).

Overall, Experiment 1 demonstrated a high degree of similarity between the two conditions following the first few occluder passages. Infants performed at asymptote from the beginning in the variable linear condition and reached compatible performance level from the third occlusion passage in the fixed non-linear condition. Since no previous occlusion studies have reported such rapid learning effects at 6 months of age it becomes important to replicate these findings. Experiment 2 presents another group of 6-month-olds with the fixed non-linear condition. In addition, Experiment 2 attempts to improve our understanding of the stability of these representations by presenting infants with multiple sessions, extending over a $24 \mathrm{~h}$ period. 


\section{Experiment 2}

The second experiment focuses on infants' performance during the fixed nonlinear condition. It had two purposes; first of all, to replicate the learning effects of Experiment 1, and second, to investigate the stability of this learning over time. This was tested by presenting infants with three identical sessions of non-linear passages; the first two are separated by $15 \mathrm{~min}$ and the last occurred $24 \mathrm{~h}$ later. No previous study has examined the effects of such prolonged intermissions on 6-month-olds representational abilities using an occlusion paradigm.

\subsection{Method}

\subsubsection{Participants}

Twenty-one infants participated in Experiment 2 (13 girls and 8 boys; mean age $27: 4$ weeks, $S D=3$ days). Each participant visited the lab on two occasions. The time interval between the two visits was $24 \mathrm{~h}$. Four infants ( 3 girls and 1 boy) were excluded from the analysis. The final sample included 17 infants, who performed according to inclusion criterion (see above) at 2 out of the 3 experimental sessions. Out of the 51 sessions presented to these infants, 44 were included in the final analysis.

\subsubsection{Stimuli and design}

Infants were presented with three sessions of 16 occlusion events. The stimuli were identical to the ones used in the fixed non-linear condition of Experiment 1 . Between the first and second session there was a 15 min break in which the infants were removed from the car seat. The time was spent feeding or playing with toys in an adjacent waiting room. After $24 \mathrm{~h}$ each family returned to the lab for a third learning session. Altogether infants were presented with 48 separate occlusion events ( 3 sessions $\times 8$ trajectories $\times 2$ occlusion passages). Following the final presentation parents were given the opportunity to view a movie of their infants tracking the target.

\subsection{Results}

\subsubsection{Overall performance}

In response to the first session 187 occlusion passages $(69 \%)$ were included in the analysis. The second session included 151 passages $(67 \%)$ and the third and final session included $128(62 \%)$ occlusion passages. Remaining passages did not pass the inclusion criteria.

Tracking patterns during these attentive passages were highly compatible with performance in Experiment 1. Infants fixated the disappearance edge until they made a predictive or reactive gaze shift in $71 \%$ of all attentive passages during the first session, $76 \%$ during the second, and $75 \%$ during the third session. The second most common response pattern involved shifting gaze from the disappearance edge to the center of the occluder before making a predictive or reactive gaze shift $(10 \%, 15 \%$, and $21 \%$ of all attentive passages in each of the three sessions). During remaining 
attentive trails $(9 \%, 9 \%$, and $5 \%$ in each of the three sessions) infants searched the rest of the screen or scanned the occluder edge without clear fixations.

The median predictive saccade occurred after the ball had been occluded for $637 \mathrm{~ms}(S E=33 \mathrm{~ms})$ during the first session, after $676 \mathrm{~ms}(S E=36 \mathrm{~ms})$ during the second session, and after $667 \mathrm{~ms}(S E=43 \mathrm{~ms})$ during the third session. Reactive saccades were initiated 1041, 1064, and $1022 \mathrm{~ms}(S E=9$, 9, and $9 \mathrm{~ms})$ after the ball became occluded. The total occlusion duration equalled $700 \mathrm{~ms}$.

Two Friedman ANOVAs for multiple dependent samples were calculated; one for prediction rate and the other for accuracy rate. In each ANOVA the dependent variable was divided into $2 \times 3$ levels, comparing the first and last half of each of the three sessions. The first Friedman ANOVA did not demonstrate any differences in prediction rate over the three sessions. Prediction rates equaled $0.5(S E=0.041)$ during the first session, $0.5(S E=0.040)$ during the second session, and $0.6(S E=0.038)$ during the third and final session and averaged $0.53(S E=0.029)$ for the first half of each session and $0.56(S E=0.038)$ for the last half (averages combined over sessions).

The second Friedman ANOVA demonstrated a significant effect of accuracy rate over successive occlusion passages, $\chi^{2}(N=11, d f=5)=12.27, p<.05$. The overall accuracy rate equaled $0.42(S E=0.1)$ and $0.71(S E=0.081)$ for the first and last half of the first session, $0.58(S E=0.071)$ and $0.84(S E=0.055)$ for the second session, and finally, $0.67(S E=0.050)$ and $0.78(S E=0.084)$ for the third session. A post-hoc comparison of the Friedman ANOVA (Siegel \& Castellan, 1988) revealed significant differences between the beginning of the first session and the end of the second session $(p<.01)$. There were also significant differences between the beginning of the first session and both the beginning and end of the third session $(p<.05)$.

\subsubsection{Within session analysis}

During the first session infants rapidly learned to predict the reappearance location of the target within a few trials. Accuracy rates equalled 0.0 during the first passage, 0.0 during the second, 0.5 during the third and 0.40 during the fourth passage. This learning effect is best described using the logarithmic function $y=1.0+29 \ln (x)$, expressing a rapid increase in accuracy rate over the first few trials (see Fig. 6 ), $R^{2}=.58, F(1,14)=19.4, p<.001$.

During the second session infants displayed a similar response pattern. Accuracy rates at the first four passages equaled $0.17,0.75,0.57$, and 0.80 , respectively. This rapid increase is best expressed by a significant linear regression effect, $R_{\text {adj }}^{2}=.45$, $F(1,14)=13.4, p<.003$, whose function, $y=43.4+3.2 x$ can also be observed in Fig. 6.

During the final session infants' performance did not change significantly, though accuracy rates during the first four passages equaled $0.45,0.73,0.54$, and 0.67 . No significant regression effect could be observed within this session.

\subsubsection{Extrapolations during each of the three sessions}

During the first passage of the first session $100 \%$ of all inaccurate predictions were made in the linear extension of the pre-occlusion trajectory. During the following 


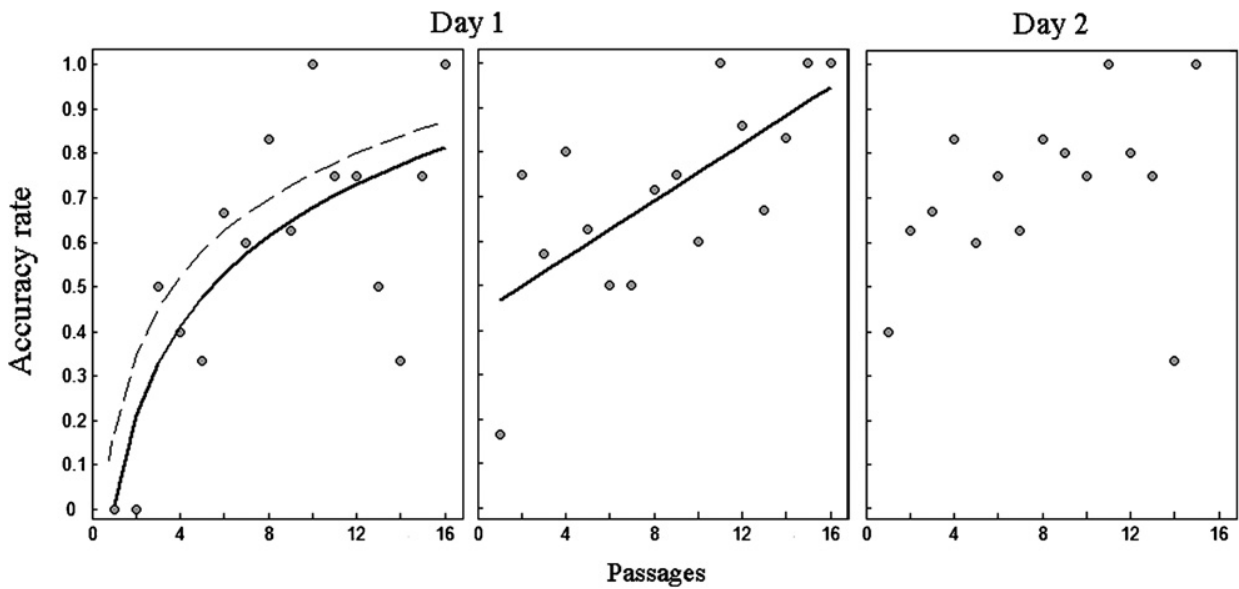

Fig. 6. Accuracy rates (number of accurate predictions/(number of accurate + inaccurate predictions)) over successive occlusion passages in each block of Experiment 2. The first graph panel of Day 1 depicts initial testing session; the second graph of Day 1 depicts second experimental session after 15 min break. Graph panel of Day 2 depicts third experimental session after $24 \mathrm{~h}$ break. The solid curve depicts the regression line with most explained variance. The dashed line shows the regression curve in the fixed nonlinear condition of the Experiment 1. Note: No significant regression line exists for the third session.

passages the tendency to extrapolate the pre-occlusion trajectory decreased $\left(R^{2}=.44, F(1,14)=10.92, p<.005\right)$. This decrease was best described using the curvilinear function $y=72-20.24 \ln (x)$ visible in Fig. 7. No significant regression effects could be observed during the second and third session.

\subsection{Discussion}

Results from the first session of Experiment 2 replicate the rapid learning effects of Experiment 1 . In both experiments the accuracy rate increased significantly over the first few occlusion passages with nearly identical regression lines. The tendency to extrapolate the non-linear trajectory also decreased at a similar rate.

As indicated by the regression lines in Fig. 6, infants' accuracy rates increased to asymptotic levels after only a few presentations in each of the first two sessions. However, performance levels dramatically decreased in the 15 min break between these two sessions. It was only during the third session that infants' performed at asymptote from the very first occlusion passage. This finding suggests that 6month-old infants have the ability to rapidly learn where an occluded object will reappear. However, these results (supported by the overall increase in accuracy rates reported by the Friedman ANOVA) also suggest that infants require some training to withstand long breaks. This effect is quite remarkable given the massive flow of visual information that infants are bombarded with during the $24 \mathrm{~h}$ in between the second and third session. In the occlusion paradigm such learning effects, extending over $24 \mathrm{~h}$, have only been reported for 14-month-old infants (Moore \& Meltzoff, 2004). 


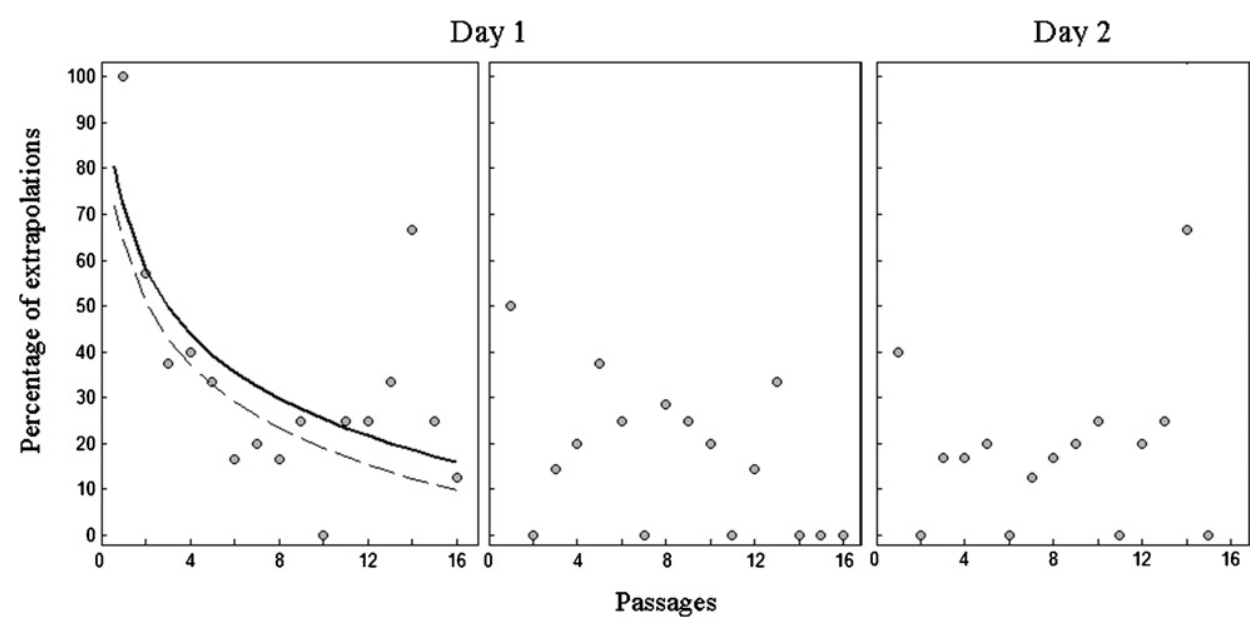

Fig. 7. Percentage of extrapolations (i.e. inaccurate predictive gaze shifts that followed the linear extension of the targets pre-occlusion trajectory) during each session of Experiment 2. The first graph panel of Day1 depicts initial testing session; the second graph of Day 1 shows second experimental session after 15 min break. Graph panel of Day 2 depicts third experimental session after $24 \mathrm{~h}$ break. The solid line represents the regression line with most explained variance. The dashed line shows the regression line for extrapolations in the fixed non-linear condition of the Experiment 1. Note: No significant regression line existed for the second and third session.

\section{General discussion}

In summary, Experiment 1 compared infants' ability to extrapolate the current pre-occlusion trajectory (variable linear condition) with their ability to base predictions on recent experiences (fixed non-linear condition). In the variable linear condition infants performed at asymptote from the first occlusion passage and continued to do so for the remainder of the session. In the fixed non-linear condition all infants initially failed to predict the reappearance location of the target. Performance, however, reached asymptote after only two occlusion passages. This is the first study that demonstrates such rapid learning effects during an occlusion task. Experiment 2 replicates these effects and demonstrates a robust memory effect extending $24 \mathrm{~h}$. In occlusion tasks such long-term memory effects have previously only been observed in 14-month-old infants (Moore \& Meltzoff, 2004).

In each of the two studies and in all conditions tested infants were equally proficient in their ability to represent the occluded object (prediction rate). These similarities are not surprising given the fact that all variables except the actual trajectory were identical (saliency of the ball and the occluder, occlusion duration, time of accretion/deletion, velocity of the target, and the amplitude of the target). Instead infants differed only in their ability to make accurate predictions (accuracy rate). In other words, infants realized, to an equal degree in each condition, that the object existed behind the occluder. What did differ was infants' ability to predict where the target would reappear. To solve this task infants' either relied on pre-existing 
assumptions or formed novel expectations based on previous events, depending on the current task demands. The following text will discuss the implications of observed commonalities in prediction rate and observed differences in accuracy rate across conditions.

\subsection{Prediction rate}

Prediction rates in this study range from 0.43 to 0.62 . This means that half of all attended passages were tracked in a reactive manner. Several aspects of this finding are worth highlighting.

First of all, it is important to emphasize that the current study, unlike previous occlusion studies, presented infants with tasks that could only be solved by relying on either recent experience or on pre-existing assumptions about how objects move (extrapolations) to solve the occlusion task. In the fixed non-linear condition of Experiment 1 infants were forced to aggregate new knowledge to solve the occlusion task. In the alternative condition (variable linear) infants could simply apply pre-existing knowledge and extrapolate the occluded object.

The vast majority of published occlusion studies present infants with multiple linear or curvilinear trajectories of objects that move back and forth behind an occluder in a repetitive manner (Gredebäck \& von Hofsten, 2004; Gredebäck et al., 2002; Johnson et al., 2003; Rosander \& von Hofsten, 2004; van der Meer, van der Weel, \& Lee, 1994). In these experiments infants tendency to extrapolate coincide with their recent experiences; both predicting the same outcome. One could therefore assume that the prediction rates would be higher in these other studies. Until now, only two occlusion studies that measure gaze tracking have reported the overall prediction rate in 6-month-old infants (Gredebäck \& von Hofsten, 2004; Johnson et al., 2003). However, prediction rates observed in those studies are highly comparable with level of prediction rate demonstrated by infants in this study. For example Johnson et al. (2003) reported prediction rates of 0.43 whereas Gredebäck and von Hofsten (2004) reported prediction rates ranging from 0.17 to 0.38 , depending on occlusion duration.

In a similar manner, infants were attentive in $73 \%$ of all occlusion passages in the variable linear condition and in between $62 \%$ and $69 \%$ of all trials in the fixed non-linear condition. These numbers are compatible to attention levels reported by Johnson et al., $2003(66-75 \%)$ and higher than the 51\% attentive trials reported by Gredebäck and von Hofsten (2004). We can therefore conclude that our infants predicted the reappearance of the target at a rate equivalent to what has previously been suggested.

\subsection{Accuracy rate}

The present study demonstrates that 6-month-olds have an initial tendency to predict the reappearance location of temporarily occluded objects by extrapolating the pre-occlusion trajectory. We suggest that this tendency is based on pre-existing 
assumptions about the physical constraints of moving objects. The fact that infants perform extrapolations throughout the entire variable linear condition, as well as during the initial non-linear passages suggests that this ability might be fundamental to how infants perceive occluded objects. This finding goes beyond previously reported abilities. von Hofsten et al. (2000) only demonstrated reliable linear predictions from the third occlusion passage and non-linear predictions from the seventh occlusion passage in 6-month-old infants.

One likely explanation for the discrepancy between current findings and previous reports relate to methodological differences between the studies. For instance, (von Hofsten et al., 2000) relied on a head tracking paradigm to measure infants' performance. In the Discussion to Experiment 1 we mention the possibility that head movements are more resistant to rapid changes (have a larger lag) than eye movements. In addition, head movements are less accurate in the space domain compared to the gaze tracking method used in the current study. Thus, even if 6-month-old infants can extrapolate the object motion from the very first occlusion event, the method employed by these authors did not pick up the actual gaze direction of infants.

The present study demonstrates that 6-month-olds have a remarkable ability to absorb information from recent events and maintain this information to predict the outcome of future occlusion events. We suggest that infants form new expectations that guide their actions by perceiving reoccurring events and generalize these events to novel situations. Infants' rapid learning in predicting non-linear trajectories suggests that this ability play an important role in how we perceive occluded objects.

Throughout this paper we have stressed the differences between the two conditions presented to infants. Linear trajectories are extrapolated whereas non-linear trajectories are predicted through an accumulation of recent experiences. However, the rapid long-term learning effects demonstrated in Section 4 indicate that these two abilities may have a common ontology.

We suggest that infant's initial tendency to extrapolate and their ability to generalize from recent experience both stem from past experience. This does not mean that there are no differences between the two conditions. Instead we argue that existing differences arise from variations in the frequency of exposure to these different constraints. That is, infant's tendency to extrapolate is closely related to the natural constraints governing object motion. This means that infants have had ample experience with these constraints. In contrast, the non-linear trajectory is governed by novel constraints that infants have little or no experience with.

To sum up, the present study demonstrates that 6-month-old infants possess a good understanding of the basic physical constraints that govern object motion. This understanding is easily generalized to novel situations like the present. As a consequence thereof infants display accurate linear predictions from the first occlusion passage. In addition, 6-month-olds have a remarkable ability to adjust their expectations to accommodate unexpected events. After being presented with only two fixed non-linear occlusion events infants adjust their predictions accordingly. At this stage infant's ability to maintain these novel expectations are easily disrupted by temporal delays. Only after multiple sessions will infants new experiences stabilize long enough to withstand a $24 \mathrm{~h}$ break. 


\section{Acknowledgements}

We thank Claes von Hofsten and researchers from Uppsala Babylab for help for comments as well as the enthusiastic parents and infants who made this research possible. The study was supported by grants to Claes von Hofsten from The Swedish Research Council, (2004-12172-22115-26), McDonnell Foundation (21002089), and European Union (EU005010: contact).

\section{References}

Baillargeon, R. (1999). Young infants' expectations about hidden objects: a reply to three challenges. Developmental Science, 2(2), 115-163.

Canfield, R. L., \& Haith, M. M. (1991). Young infants' visual expectations for symmetric and asymmetric stimulus sequences. Developmental Psychology, 27, 198-208.

Engel, K. C., Anderson, J. H., \& Soechting, J. F. (1999). Oculomotor tracking in two dimensions. Journal of Neurophysiology, 81, 1597-1602.

Gredebäck, G., \& von Hofsten, C. (2004). Infants' evolving representations of object motion during occlusion: a longitudinal study of 6- to 12-month-old infants. Infancy, 6(2), 165-184.

Gredebäck, G., von Hofsten, C., \& Boudreau, J. P. (2002). Infants' visual tracking of continuous circular motion under conditions of occlusion and non-occlusion. Infant Behavior \& Development, 25(2), $161-182$.

Gredebäck, G., Örnkloo, H., \& von Hofsten, C. (2006). The development of reactive saccade latencies. Experimental Brain Research, 173(1), 159-164.

Johnson, S. P., Amso, D., \& Slemmer, J. A. (2003). Development of object concepts in infancy: evidence for early learning in eye-tracking paradigm. Proceedings of the National Academy of Sciences of the United States of America, 100(18), 10568-10573.

Haith, M. M. (1994). Visual expectations as the first step toward the development of future-oriented processes. In M. M. Haith, J. B. Benson, R. J. Roberts, \& B. F. Pennington (Eds.), The development of future-oriented processes (pp. 11-38). Chicago: University of Chicago Press.

Hartshorn, K., Rovee-Collier, C., Gerhardstein, P. C., Bhatt, R. S., Wendoloski, T. L., Klein, P., et al. (1998). Developmental changes in the specificity of memory over the first year of life. Developmental Psychobiology, 33(1), 61-78.

Hartshorn, K., \& Rovee-Collier, C. (1997). Infant learning and long-term memory at 6 months: a confirming analysis. Developmental Psychobiology, 30(1), 71-85.

Hitchcock, D. F. A., \& Rovee-Collier, C. (1996). The effect of repeated reactivations on memory specificity in infancy. Journal of Experimental Child Psychology, 62(3), 378-400.

Mareschal, D. (2000). Object knowledge in infancy: current controversies and approaches. Trends in Cognitive Sciences, 4(11), 408-416.

Meichler, M., \& Gratch, G. (1980). Do 5-month-olds show object conception in Piaget's sense?. Infant Behavior and Development 3, 256-281.

Moore, M., \& Meltzoff, A. N. (2004). Object permanence after a 24-h delay and leaving the locale of disappearance: the role of memory, space, and identity. Cognition, 40(4), 606-620.

Munakata, Y. (1998). Infant perseveration and implications for object permanence theories: a PDP Model of the ab task. Developmental Science, 1(2), 161-184.

Nelson, K. E. (1971). Accommodation of visual-tracking patterns in human infants to object movement patterns. Journal of Experimental Child Psychology, 12, 182-196.

Nelson, K. E. (1974). Infants' short-term progress towards one component of object permanence. MerrilPalmer Quarterly, 20, 3-8.

Piaget, J. (1952). The origins of intelligence in children. New York: International University Press.

Rosander, R., \& von Hofsten, C. (2004). Infants' emerging ability to represent object motion. Cognition, 91, 1-22. 
Siegel, S., \& Castellan, N. J. (1988). Nonparametric statistics for behavioral sciences (2nd ed.). New York: McGraw-Hill Book Co..

Spelke, E. S., Breinlinger, K., Macomber, J., \& Johnson, K. (1992). Origins of knowledge. Psychological Review, 99, 605-632.

Spelke, E. S., Katz, G., Purcell, S. E., Ehrlich, S. M., \& Breinlinger, K. (1994). Early knowledge of object motion: continuity and inertia. Cognition, 51, 131-176.

Spelke, E. S., \& von Hofsten, C. (2001). Predictive reaching for occluded objects by six-month-old infants. Journal of Cognition and Development, 2, 261-282.

van der Meer, A. L. H., van der Weel, F., \& Lee, D. N. (1994). Prospective control in catching by infants. Perception, 23, 287-302.

von Hofsten, C., Feng, Qi., \& Spelke, E. S. (2000). Object representation and predictive action in infancy. Developmental Science, 3(2), 193-205.

Wilcox, T., Rosser, R., \& Nadel, L. (1994). Representation of object location in 6.5-month-old infants. Cognitive Development, 9(2), 193-209. 